NOTULE HYDRAULIQUE HYDRAULIC BRIEF

\title{
Sur les élargissements brusques de petite amplitude
}

\section{Small, sudden increases in section}

\begin{abstract}
Ponr l'évaluation de la pression régnant autour d'un jet dans un élargissement brusque, la théorie classique est en contradiction avec celle de la diffusion du jet. Mais cette contradiction n'a qu'une incidence pratiquement négligeable sur la perte de charge singulière dans l'élargissement.
\end{abstract}

On sait que la théorie classique de l'élargissement brusque dans une conduite en charge est fondée sur l'hypothèse que, dans toute section élargie, au droit de l'élargissement, il règne la même pression que dans le jet. Cela revient à admettre qu'autour du jet se trouve de l' " eau morte $»$; or, c'est en contradiction avec la théorie de la diffusion du jet qui prévoit un appel d'eau prise dans la zone entourant le jet pour s'y mélanger par le mécanisme de la turbulence. On est donc conduit à penser que ce phénomène doit fausser la théorie classique et probablement d'autant plus que la zone entourant le jet est plus restreinte; mais, objectera-t-on, cet effet doit se manifester par une divergence entre la perte de charge réelle et celle que l'on peut calculer par la formule généralement admise :

$$
\Delta h=\frac{\left(\mathrm{V}_{0}-\mathrm{V}_{1}\right)^{2}}{2 g}
$$

Il semble que, jusqu'ici, elle n'ait pas été constatée expérimentalement. Cela s'explique: d'une part, les auteurs se sont probablement peu intéressés aux élargissements de petite amplitude; d'autre part, nous allons montrer que, si cette divergence existe, elle est difficilement décelable par des mesures de perte de charge, car une différence éventuelle entre la pression moyeune $p$ autour du jet et la pression $p_{0}$ dans le jet ne se traduit, quand l'élargissement est faible, que par une différence du second ordre sur la perte de charge :

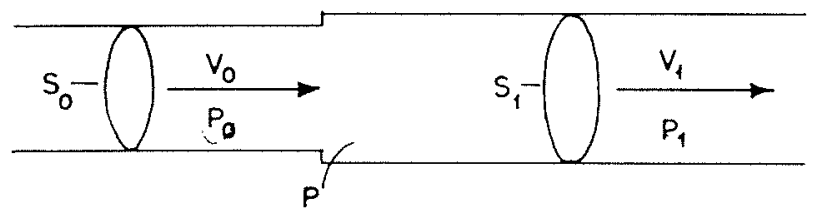

When estimating the pressure around a jet al a sudden increase in the section, standard theory is in contradiction with that concerning the diffusion of a jet. However this contradiction has a practically negligible effect upon the head loss which occurs at this point.

L'équation des quantités de mouvement, abstraction faite des forces tangentielles, s'écrit :

$\rho \mathrm{SV}_{1}\left(\mathrm{~V}_{0}--\mathrm{V}_{1}\right)=\mathrm{S}_{1} p_{1}-\mathrm{S}_{0} p_{0}-\left(\mathrm{S}_{1}-\mathrm{S}_{0}\right) p$,

d'où :

$$
p_{1}-p_{0}=\rho V_{1}\left(V_{0}-V_{1}\right)+\frac{S_{1}-S_{0}}{S_{1}}\left(p-p_{0}\right),
$$

au lieu de :

$$
\begin{aligned}
p_{1}-p_{0}=\rho & \mathrm{V}_{1}\left(\mathrm{~V}_{0}-\mathrm{V}_{1}\right), \\
& \text { dans l'hypothèse où } p=p_{0} .
\end{aligned}
$$

Le terme complémentaire se traduit sur la perte de charge par la différence :

$$
\Delta \Delta h=\frac{p-p_{0}}{\rho g} \frac{\mathrm{S}_{1}-\mathrm{S}_{0}}{\mathrm{~S}_{1}} .
$$

Pour des élargissements de plus en plus petits :

$$
\frac{S_{1}-S_{0}}{S_{1}} \rightarrow 0
$$

donc $\Delta \Delta h$ est bien du second ordre par rapport à $p-p_{0}$.

Enfin, la définition même de la perte de charge singulière perd toute netteté dans la pratique, à cause de la perte de charge répartie due à la rugosité, particulièrement dans le cas qui nous occupe. Voir sur ce sujet la note de M. P. AlméRAS : $3^{\circ}$ Réunion A.I.R.T.H., Grenoble, septembre 1949, communication $\mathrm{n}^{\circ}$ III, 5 .

R. LEMOINE. Ingénieur à la Sogréah, Grenoble. 